\title{
Pulmonary Function of Petrol filling Workers from West Bengal, India
}

\author{
Mandal (Majee) A $\mathbf{A}^{1}$, Mukherjee $\mathbf{N}^{2}$
}

1'Department of Physiology, Raja Peary Mohan College, University of Calcutta, Hooghly, West Bengal, India ${ }^{2}$ Department of Physiology, Serampore College, Serampore, West Bengal, India

\section{ABSTRACT}

Introduction: There are many petrol filling stations in urban and semi urban areas of Hooghly District of West Bengal and a large number of attendants work in these stations. They are constantly exposed to petrol vapour as well as motor vehicle fuel which might lead to respiratory problems in workers.

Objectives: To assess the pulmonary function of Petrol Filling Workers of West Bengal and to evaluate the effect of duration of exposure on those Parameters.

Methods: This is a cross-sectional study design. Pulmonary function test was done among 52 Petrol filling workers and 20 office workers of college as well as students of those institution of Hooghly district and nearby area of Kolkata by simple random sampling method. Pulmonary function parameters were assessed by digital expirograph (Spirovit SP1) and Wright's Peak Flow Meter. A questionnaire was filled up by invigilator regarding years of exposure, respiratory abnormalities, smoking habit and any other diseases. All the study protocol was approved by Human ethical Committee of Raja Peary Mohan College and Serampore college

Results: From the above survey it was observed that respiratory volume and capacities significantly $(p<0.05-0.001)$ decreased with increase in years of exposure. Odd ratios of different respiratory diseases (OR ranges from 1.123.12) indicate risk of developing cough, phlegm, asthma, skin diseases among petrol filling workers.

Conclusion: Safe threshold level of volatile organic compounds VOC exposed in Petrol station is needed to protect the health of workers. Besides periodic health check-up, use of protective gadgets, improvement of technology and control strategies to reduce air emission of VOC-is essential to protect the health of Petrol filling workers of West Bengal.

Key words: Odds Ratio, Petrol filling Workers, Prevalence of respiratory diseases, Pulmonary function,

DOI: https://doi.org/10.3126/ijosh.v10i1.29879

\section{Introduction}

etrol, also called gasoline is a complex combination of hydrocarbon. Petrol vapour contains 95\% aliphatic and cyclic compounds and less than $2 \%$ are aromatic compounds. ${ }^{1}$ Benzene content of petrol has typically been in the range of $1-5 \%$. Typical average for 8 hour benzene exposure in distribution and retail

Date of submission: 11.06.2020

Date of acceptance: 11.07.2020

\section{Corresponding Author}

Anindita Mandal (Majee), M.Sc, Ph .D

Associate Professor

Department of Physiology

Raja Peary Mohan College, University of Calcutta

P.O-Uttarpara Dist-Hooghly, Pin-712258

West Bengal, India

Phone: 033- 2663-0881/ 033-2663-6745/9433910161

E-mail: anindita11us@rediffmail.com operations is less than $1 \mathrm{ppm}$ (parts per million) although exposures can reach 2-3 ppm for shorter periods. $^{1}$

Petrol filling station is a place where workers are exposed to both petroleum vapours and vehicular exhaust. The effects of petrol vapours and the suspended particulate matter may affect of the lung function. ${ }^{2}$

In India, Petrol filling workers are employed for fuel loading rather than self-service which can cause exposure to petrol vapour. Long term exposure to petrol vapour has shown to affect the different physiological systems in the body. ${ }^{3}$

\section{(c) (i) (8)}

This journal is licensed under a Creative Commons AttributionNon Commercial 4.0 International License. 
Air pollutants and chemicals like Benzene, lead, other heavy metals, carbon monoxide and their metabolites can cause adverse health effects by interacting with molecules crucial to the biochemical or physiological process of the human body. ${ }^{4}$ All these have been found to lead to deleterious effect on respiratory, endocrine and haemopoietic systems. High ambient air concentration of solvents and pollutants had well defined and marked systemic pulmonary inflammatory response with decreased FVC (Forced Vital Capacity), $\mathrm{FEV}_{1}$ (Forced expiratory volume in $1 \mathrm{sec}$ ), Inspiratory and expiratory flow rates. ${ }^{5}$

There are many Petrol filling stations in urban and semi urban area of Hooghly District of West Bengal and a large number of attendants are working in these stations. They are constantly exposed to petrol vapour as well as motor vehicle fuel which might lead to health problem in workers. Therefore, we conducted this study to evaluate the changes in pulmonary function tests of Petrol filling workers in West Bengal. Therefore, the objectives of the present study is -

1. To assess the pulmonary function of petrol filling workers who were exposed to petrol and diesel as well as control group.

2. To evaluate the effect of duration of service/ exposure at the petrol pump on the degree of derangement in pulmonary function.

\section{Methods}

This cross-sectional study was conducted in the Petrol filling stations of different areas of West Bengal. Study on Control group was made in their work place and department of physiology of Raja Peary Mohan College and Serampore College. The study group comprised of 52 male Petrol filling workers and 20 control group individuals of same age group male adults working as office workers of college and students of the same area during September - October 2018 by simple random sampling method.

\section{Study Area}

The study area has been divided in 4 sub areas like - Kolkata area, Uttarpara area, Bhadrakali area and Bally area. The subjects were selected at random from petrol pump of different areas.

\section{Inclusion Criteria}

- Subjects working for more than 1 year in the Petrol Station

\section{Exclusion Criteria}

- Workers working for less than 1 year in the petrol filling station.

- Workers with history of tuberculosis, emphysema, and Diabetes mellitus

- Workers with clinical abnormalities of vertebral column \& the thorax were excluded.

\section{Respiratory Health Questionnaire}

A questionnaire was filled up by the investigator. A modified version of the British Medical Research Council questionnaire ${ }^{6}$ was used for respiratory health questions which include information regarding age, smoking habit, feeling of any respiratory distresses during daily activities, job description .

\section{Ethical Consideration}

The study protocol was first approved by human Ethical Committee of Raja Peary Mohan College and Serampore College. Besides this, consent from manager of Petrol stations and college authorities as well as participants were taken after explaining the purpose of this study.

The pulmonary function tests (PFT) were performed in their workplace by using automatic spirometer (Spirovit SP 1 model) according to the guideline recommended by American thoracic society. ${ }^{7}$ The testing procedures are quite simple and non-invasive and harmless to the participants. The spirometer was calibrated each day prior to use and a new filter was used. The relevant data- age, sex, body weight was recorded. Proper instructions were given to the subject in order to familiarize himself with the equipment. During the tests the subjects were adequately encouraged to perform their optimum level and also a nose clip was applied. The test was repeated 3 times and best results were considered for analysis.

\section{Pulmonary Function Parameters Studied}

Forced vital capacity (FVC), Force expiratory volume in $1 \mathrm{sec}$ (FEV1),

Slow vital capacity (SVC), Expiratory reserve volume (ERV), Inspiratory reserve volume (IRV), Tidal volume (TV), FEV1/SVC, FEF 25\%-75\%, Maximum voluntary ventilation (MVV), FEF $0.2-1.2 \%$, FEF $75 \%-85 \%$ and PEFR

PEFR is measured by Wright's Peak Flow Meter. The 
subject was instructed to take a deep breath and place the mouth piece in his/her mouth, between the teeth and with the lips placed tightly around it. Then, they are asked to blow in to the instrument with a short, sharp blow. Reading was recorded. The pointer was reset to zero, by pressing the release button. After a couple of practice blows, 3 attempts in succession were taken and all three attempts were recorded. It is expressed by lit/min.

All the tests were performed between 2 PM to 4 PM to exclude the bias of circadian rhythm. All the gas volumes were corrected to BTPS (Body temperature, ambient pressure and saturated with water vapour) automatically by the instrument.

\section{Physical and Cardiovascular Parameters}

Age was recorded from the office record.

Body height was measured by anthropometric rod.

Body weight was taken by using weighing machine with the participant wearing light clothing and without shoes.

Body mass index (BMI) was calculated using formulae Body weight in $\mathrm{Kg} /$ (body height in meter) ${ }^{2}$

Blood pressure was measured by auscultatory method with the help of a Sphygmomanometer and Stethoscope.

Heart rate was taken by palpatory method.

\section{Results}

Social demographic characteristics of the sample population - this study covered a total of 72 respondents including 54 petrol filling workers (PFW) and 20 office workers. This means the PFW comprised of $72 \%$. while office workers make $28 \%$ of the total number of respondent. The age distribution, working period, smoking habit are present in Table 1.

Table 2 represents mean \pm SD values of age, body height, body weight, BMI, blood pressure and heart rate values of petrol filling workers (PFW) and control group(unexposed) workers. No significant difference has been found in age, heart rate and diastolic blood pressure values between petrol filling workers and control group worker. Body weight, BMI and systolic blood pressure is significantly higher in PFW $(\mathrm{P}<0.05-$ 0.01 ) than control group workers.
Table 3 represents mean \pm SD values of different pulmonary function parameters of Petrol filling workers and control group. All the pulmonary function parameters are significantly lower than control group (unexposed) workers except TV, FEV $1, \mathrm{FEF}_{25-75 \%}$ and FEF $_{75-85 \%}$.

Table 4 represents mean \pm SD values of physical heart rate, blood pressure and pulmonary function parameters of lower and higher age group PFW. It is found that blood pressure and heart rate values are significantly higher in higher age group PFW but most of the pulmonary function parameters are significantly higher $(p<0.05-0.0001)$ in lower age group PFW workers than higher age group.

Table 5 represents mean $\pm S D$ values of physical parameters, heart rate, blood pressure and pulmonary function parameters on the basis of year of exposure. It is found that age, height, SVC, TV, $\mathrm{FEF}_{25-75 \%}, \mathrm{FEF}_{75-}$ $85 \%$, values are significantly lower in 11-20 years of exposure in comparison to $<10$ years of exposure. It is also found that age, blood pressure (systolic), ERV, TV, FEF $25-75 \%, \mathrm{FEF}_{75-85 \%}$, MVV,PEFR values are significantly $(p<0.05-0.001)$ lower in $>20$ years of exposure in comparison to $<10$ years and $11-20$ years of exposure.

Table 6 represents comparison of mean \pm SD values of smoker and non-smoker pulmonary function Parameters with control group. It is found that both smoker and non-smoker PFW have significantly lower values of SVC, FVC, FEV , , FEF $_{0.2-1.2} \mathrm{FEF}_{25-75 \%}$ and $\mathrm{FEF}_{75-85 \%,}$ MVV and PEFR than control group.

Table 7 shows correlation values between age, height, weight, BMI, blood pressure and heart rate and year of exposure with pulmonary function parameters. It is found that all the pulmonary function parameters are significantly correlated with age, body height, body weight and BMI except SVC.MVV was significantly associated $(p<0.05)$ with age and height. All the pulmonary function values showed significantly $(p<0.05-0.001)$ but negative correlation with age except

SVC, TV and FVC ( $>00.05)$, Highly significant correlation has been found $(p<0.05-0.001)$ between year of exposure and pulmonary function parameters.

Table 8 represents the percentage prevalence of respiratory abnormalities among PFW in comparison to control group. It was observed that $30.76 \%$ PFW had restrictive lung impairment, $23.07 \%$ and $11.53 \%$ had 
obstructive and combined lung impairment respectively in comparison to $5 \%$ restrictive, $10 \%$ obstructive and none had combined lung impairment in control group workers.

It revealed that petrol filling workers who smoke were more prone to have prevalence of chest tightness, asthma and skin problem compared to non-smoking workers.(table 9)

It was observed that higher age group petrol Filling workers were more prone to Chest tightness, asthma and skin problem in comparison to lower age group.

(Table 10)
It was found that workers of $>20$ years of exposure were more to cough, Phlegm and asthma than $<10$ years and 11-20 years of exposure. (table11)

Figure 1 represents the pie diagram showing percentage prevalence of respiratory diseases and other health problem among petrol filling workers. It was found that $25 \%$ (no-13) workers reported eye irritation, 21\%(N11) reported skin problem, $13 \%(\mathrm{~N} 6.7=7)$ reported asthma and Phlegm, (17\%, N 9) reported cough and $11 \%(\mathrm{~N} 6)$ reported chest tightness.

Table 1: Social demographic characteristics

\begin{tabular}{|c|c|c|}
\hline Characteristics & $\begin{array}{l}\text { Petrol filling workers } \\
\qquad n=52\end{array}$ & $\begin{array}{l}\text { College/office workers } \\
\qquad n=20\end{array}$ \\
\hline \multicolumn{3}{|l|}{ Age group } \\
\hline 18-34 years & $48.07 \%$ & $65 \%$ \\
\hline$>34$ years & $51.92 \%$ & $35 \%$ \\
\hline \multicolumn{3}{|l|}{ Working period } \\
\hline $0-10$ years & $57.69 \%$ & $25 \%$ \\
\hline $11-20$ years & $15.38 \%$ & $50 \%$ \\
\hline$>20$ years & $26.92 \%$ & $25 \%$ \\
\hline \multicolumn{3}{|l|}{ Smoking habit } \\
\hline Yes & $53.84 \%$ & $35 \%$ \\
\hline No & $46.15 \%$ & $65 \%$ \\
\hline \multicolumn{3}{|c|}{ Daily working hours } \\
\hline 8 hours or less & $42.85 \%$ & $60 \%$ \\
\hline$\geq 8$ hours & $57.14 \%$ & $40 \%$ \\
\hline \multicolumn{3}{|c|}{ Use of personal protective equipment } \\
\hline Yes & Nil & nil \\
\hline No & $100 \%$ & $100 \%$ \\
\hline
\end{tabular}

Table 2: Mean \pm SD values of Physical and some Physiological Parameters of Petrol filling workers (PFW) and control group

\begin{tabular}{|c|c|c|c|}
\hline Parameters & PFW & control & $t$ test \\
\hline AGE (yrs) & $35.91+\_11.78$ & $30.7+12.59$ & 0.12 \\
\hline $\mathrm{HEIGHT}(\mathrm{cm})$ & $164.6+6.18$ & $167.9+4.5$ & 0.019 ** \\
\hline WEIGHT (kg) & $66.6+12.4$ & $61.37+8.78$ & $0.05^{*}$ \\
\hline BP(systolic) (mm Hg) & $139.3+\ldots 18.8$ & $129.4+13.1$ & $0.01^{* * *}$ \\
\hline BP(Diastolic) (mmHg) & $79.7+11.5$ & $77.4+99.6$ & 0.39 \\
\hline HEART RATE (beats/min) & $83.1+11.96$ & $79.45+6.53$ & 0.10 \\
\hline BMI $\left(\mathrm{Kg} / \mathrm{m}^{2}\right)$ & $24.52+4.3$ & $21.84 \pm 3.82$ & $0.01^{* * *}$ \\
\hline
\end{tabular}

$\mathrm{P}<0.05-{ }^{*}, \mathrm{p}<0.02^{* *}, \mathrm{p}<0.01$ - $^{* * *} \mathrm{BMI}=$ Body Mass Index 
Mandal (Majee) A et al.

Table 3: Mean $\pm S D$ values of different pulmonary functional parameters of Petrol filling workers (PFW) and control group.

\begin{tabular}{|c|c|c|c|}
\hline Respiratory Parameters & $\begin{array}{c}\text { PFW } \\
(n=52)\end{array}$ & $\begin{array}{l}\text { control } \\
(n=20)\end{array}$ & $t$ test \\
\hline SVC & $2.66+0.64$ & $4.2+0.93$ & $0.001^{* * * *}$ \\
\hline ERV & $0.554+0.39$ & $1.2+0.69$ & $0.001^{* * * *}$ \\
\hline IRV & $1.28+0.45$ & $2.1+0.79$ & $0.0001^{* * * *}$ \\
\hline TV & $1.05+0.53$ & $0.88+0.34$ & 0.11 \\
\hline FVC & $2.70+0.78$ & $4.2+0.87$ & $0.001^{* * * *}$ \\
\hline FEV1 & $2.74+0.88$ & $3.4+\ldots 66$ & $0.001^{* * * *}$ \\
\hline FEV1/SVC & $89.5+22.29$ & $85.05+10.04$ & 0.25 \\
\hline FEF.2-1.2 & $5.15+2.04$ & $7.2+2.29$ & $0.001^{* * * *}$ \\
\hline FEF25-75\% & $4.07+-1.08$ & $4.6+2$ & 0.30 \\
\hline FEF75-85\% & $2.22+1.09$ & $2.3+\ldots 1.4$ & 0.83 \\
\hline MVV & $95.3+\_35.9$ & $153.3+37.28$ & $0.001^{* * * *}$ \\
\hline PEFR & $361+\_97.21$ & $407.7+566.64$ & $0.01^{* * *}$ \\
\hline
\end{tabular}

$p<0.05-{ }^{*}, p<0.02-{ }^{* *}, p<0.01-{ }^{* * *}, p<0.001-* * * *$

Table 4: Mean \pm SD values of physical parameters, Blood pressure, Heart rate \& pulmonary function values of higher and lower age group of petrol filling workers.

\begin{tabular}{|c|c|c|c|}
\hline & AG & & T test \\
\hline & 14-34 YEAR $(n=25)$ & 34-above $(n=27)$ & \\
\hline HEIGHT & $164.7 \pm 4.1$ & $164.7 \pm 7.6$ & 0.99 \\
\hline WEIGHT & $65.4 \pm 11.4$ & $68.0 \pm 13.6$ & 0.41 \\
\hline BP(SYSTOLIC) & $129.8 \pm 12.7$ & $149.1 \pm 19.2$ & $0.0001^{* * * *}$ \\
\hline BP(DIASTOLIC) & $77 \pm 8.8$ & $82.6 \pm 13.25$ & 0.07 \\
\hline HEART RATE & $78.6 \pm 10.9$ & $87.2 \pm 11.3$ & $0.008^{* * *}$ \\
\hline BMI & $24 \pm 3.6$ & $25.1 \pm 4.9$ & 0.35 \\
\hline SVC & $2.79+0.70$ & $2.51+0.58$ & 0.129 \\
\hline ERV & $0.49+0.41$ & $0.59+0.36$ & 0.36 \\
\hline IRV & $1.42+0.43$ & $1.16+0.43$ & $0.001^{* * *}$ \\
\hline TV & $1+0.5$ & $1.10+0.51$ & 0.06 \\
\hline FVC & $2.7+0.74$ & $2.5+0.8$ & $0.01^{* *}$ \\
\hline FEV1 & $2.6+0.81$ & $2.2+0.91$ & $0.05^{\star}$ \\
\hline FEV1/SVC & $90.4+15.6$ & $87.9+27.1$ & 0.60 \\
\hline FEF.2-1.2 & $5.7+\_1.8$ & $4.4+22$ & $0.05^{*}$ \\
\hline FEF25-75\% & $4.8+\_1.8$ & $3.3+\ldots 1.3$ & $0.001^{* * *}$ \\
\hline FEF75-85\% & $2.8+\_1.08$ & $1.6+0.7$ & $0.001^{* * * *}$ \\
\hline MVV & $105.8+\_38.3$ & $84.9+\_30.35$ & $0.03^{*}$ \\
\hline PEFR & $383.7 \pm 95.7$ & $344.2 \pm 96.5$ & $0.01^{* *}$ \\
\hline
\end{tabular}

$\mathrm{P}<0.05-{ }^{*}, \mathrm{p}<0.01-^{* *}, \mathrm{p}<0.001-{ }^{* * *}, \mathrm{p}<0.0001-{ }^{* * *} \mathrm{BMI}=$ Body Mass Index

Table 5: Comparison of Mean \pm SD values of physical parameters, heart rate, blood pressure and pulmonary function parameters of petrol filling workers based on year of exposure.

\begin{tabular}{|c|c|c|c|c|c|}
\hline & $\begin{array}{c}0-10 \\
\text { years }(n=30)\end{array}$ & $\begin{array}{c}11-20 \\
\operatorname{years}(n=8)\end{array}$ & $>20$ years $(n=14)$ & $\begin{array}{c}T \text { test }(0-10 \text { with } \\
10-20)\end{array}$ & $\begin{array}{c}T \text { test }(0-10 \text { with } \\
>20)\end{array}$ \\
\hline AGE & $29.9 \pm 9.88$ & $37.5 \pm 3.83$ & $49 \pm 6.1$ & $0.001^{* * *}$ & $0.001^{* * * *}$ \\
\hline HEIGHT & $164.6 \pm 4.5$ & $168.3 \pm 2.7$ & $163.1 \pm 9.4$ & $0.02^{*}$ & 0.61 \\
\hline WEIGHT & $66.43 \pm 12.89$ & $71.58 \pm 10.4$ & $64.6 \pm+12.4$ & 0.318 & 0.68 \\
\hline BP(systolic) & $133.2 \pm 14.9$ & $144.3 \pm 17.3$ & $151.3 \pm 21.9$ & 0.188 & $0.01^{* *}$ \\
\hline
\end{tabular}


Table 5 cont ...

\begin{tabular}{lccccc} 
BP(diastolic) & $78.2 \pm 10.2$ & $78.3 \pm 20.79$ & $83.7 \pm 8.14$ & 0.994 & 0.07 \\
HEART RATE & $78.2 \pm 10.35$ & $89.1 \pm 8.13$ & $84.8 \pm 9.9$ & 0.076 & 0.32 \\
BMI & $24.43 \pm 4.26$ & $25.32 \pm 4$ & $24.3 \pm 4.8$ & 0.638 & 0.95 \\
SVC & $2.6 \pm 0.55$ & $3 \pm 1.14$ & $2.4 \pm 0.5$ & $0.05^{*}$ & 0.25 \\
ERV & $0.5 \pm 0.4$ & $0.40 \pm 0.31$ & $.71 \pm .36$ & 0.465 & $0.01^{* *}$ \\
IRV & $1.3 \pm 0.44$ & $1.35 \pm 0.42$ & $1.1 \pm .49$ & 0.89 & 0.36 \\
TV & $1 \pm 0.5$ & $1.4 \pm 0.52$ & $0.92 \pm 0.49$ & $0.01^{* *}$ & $0.05^{*}$ \\
FVC & $2.6 \pm 0.71$ & $3.09 \pm 1.07$ & $2.73 \pm 0.72$ & 0.368 & 0.06 \\
FEV1 & $2.5 \pm 0.77$ & $2.8 \pm 1$ & $2.1 \pm 1$ & 0.49 & 0.200 \\
FEV1/SVC & $88.8 \pm 17.8$ & $97.4 \pm 28$ & $87.4 \pm 29.1$ & 0.48 & 0.873 \\
FEF.2-1.2 & $5.4 \pm 1.7$ & $4.9 \pm 2.8$ & $4.6 \pm 2.3$ & 0.71 & 0.32 \\
FEF25-75\% & $4.4 \pm 1.66$ & $3.6 \pm 2.4$ & $3.4 \pm 1.5$ & $0.02^{*}$ & $0.05^{*}$ \\
FEF75-85\% & $2.5 \pm 1.1$ & $2 \pm .68$ & $1.5 \pm 0.89$ & $0.016^{* *}$ & $0.001^{* * *}$ \\
MVV & $95.56 \pm 36$ & $114.7 \pm 53.9$ & $85.7 \pm 22.9$ & 0.43 & $0.001^{* * *}$ \\
PEFR & $371.6 \pm 96.8$ & $365 \pm 110$ & $334.6 \pm 94.7$ & 0.89 & $0.05^{*}$ \\
\hline
\end{tabular}

$\mathrm{P}<0.05-{ }^{*}, \mathrm{p}<0.01-^{* *}, \mathrm{p}<0.001-{ }^{* * *}, \mathrm{p}<0.0001{ }^{* * * *} \mathrm{BMI}=$ Body Mass Index

Table 6: Comparison of mean \pm SD values of pulmonary function parameters of smoker and non smoker petrol filling workers with control group

\begin{tabular}{|c|c|c|c|c|c|c|}
\hline \multirow{2}{*}{$\begin{array}{l}\text { Respiratory } \\
\text { parameter }\end{array}$} & \multicolumn{2}{|c|}{ Petrol filling workers } & \multicolumn{2}{|c|}{ Control } & \multicolumn{2}{|c|}{ t test } \\
\hline & $\begin{array}{c}\text { smoker } \\
(n=28)\end{array}$ & $\begin{array}{c}\text { non-smoker } \\
(n=24)\end{array}$ & $\begin{array}{c}\text { smoker } \\
(n=7)\end{array}$ & $\begin{array}{c}\text { non smoker } \\
(n=13)\end{array}$ & smoker $\mathrm{t}$ test & $\begin{array}{c}\text { non-smoker } \\
\text { t test }\end{array}$ \\
\hline SVC & $2.7+0.68$ & $2.55+0.68$ & $3.8+1.2$ & $4.57+0.68$ & $0.05^{*}$ & $0.001^{* * * *}$ \\
\hline ERV & $0.52+0.41$ & $0.56+0.42$ & $0.68+0.34$ & $1.5+0.49$ & 0.31 & $0.001^{* * * *}$ \\
\hline IRV & $1.42+0.42$ & $1.18+0.49$ & $2.26+\_1.03$ & $2.06+0.67$ & 0.07 & $0.0005^{* * * *}$ \\
\hline TV & $0.99+0.50$ & $1.10+0.56$ & $0.91+0.36$ & $0.86+0.34$ & 0.66 & $0.012^{* *}$ \\
\hline FVC & $2.64+0.82$ & $2.68+0.74$ & $3.9+1.04$ & $4.42+0.75$ & $0.001^{* * * *}$ & $0.001^{* * * *}$ \\
\hline FEV1 & $2.48+0.91$ & $2.3+0.43$ & $3.19+0.97$ & $3.6+0.38$ & $0.01^{* * *}$ & $0.001^{* * * *}$ \\
\hline FEV1/SVC & $88.39+222.49$ & $90.9+27.1$ & $84.6+\_11.39$ & $85.3+99.7$ & $0.05^{*}$ & 0.47 \\
\hline FEF.2-1.2 & $5.20+2.05$ & $4.9+2.1$ & $6.7+3.09$ & $7.5+\_1.8$ & 0.024 & $0.0005^{* * * *}$ \\
\hline FEF25-75\% & $4.12+\ldots 1.7$ & $3.9+\_1.7$ & $3.5+2.9$ & $5.9+\_1.75$ & $0.05^{*}$ & 0.08 \\
\hline FEF75-85\% & $2.25+1$ & $2.5+\_1.15$ & $1.8+\_1.4$ & $2.5+\_1.39$ & $0.05^{*}$ & $0.03^{*}$ \\
\hline MVV & $99.67+32.62$ & 85.04+_37.3 & $153.3+49.3$ & $153.3+\_31.3$ & $0.028^{* *}$ & $0.001^{* * * *}$ \\
\hline PEFR & $374.23 \pm 100.2$ & $340.86 \pm 103.8$ & $411.4 \pm 49.13$ & $405.6 \pm 62.1$ & $0.01^{* * *}$ & $0.02^{* *}$ \\
\hline
\end{tabular}

$P<0.05-{ }^{*}, p<0.01-^{* *}, p<0.001-{ }^{* *}, p<0.0001-* * * *$

Table 7: Correlation between physical parameters, cardio vascular and respiratory parameters of petrol filling workers of West Bengal

\begin{tabular}{lcccccccc}
\hline & Age & Height & Weight & BMI & BP(systolic) & BP(diastolic) & HR & $\begin{array}{c}\text { Year of } \\
\text { exposure }\end{array}$ \\
\hline SVC & -1.78 & .28 & 0.79 & 0.96 & -1.39 & -0.36 & 0.43 & $3.26^{* * *}$ \\
ERV & $3.06^{* * *}$ & $2.35^{*}$ & -0.36 & -1.78 & $-2.43^{* *}$ & 0.02 & $-2.17^{*}$ & $4.55^{* * * *}$ \\
\hline IRV & $-2.25^{*}$ & 1.78 & $2.02^{*}$ & $-2.02^{*}$ & -1.54 & 1.39 & 0.14 & $2.63^{* *}$ \\
TV & 0.21 & -0.21 & $2.25^{*}$ & $3.06^{* * *}$ & 0.94 & 0.60 & $2.02^{*}$ & $3.78^{* * * *}$ \\
FVC & -1.71 & 1.85 & 0.5 & $-2.10^{*}$ & -0.80 & 0.7 & 0.07 & $3.98^{* * * *}$ \\
FEV1 & $-3.52^{* * * *}$ & $2.50^{* *}$ & 0.69 & $-2.43^{* *}$ & $-3.21^{* * *}$ & -0.57 & 0.073 & $2.30^{*}$ \\
\hline FEV1/SVC & $-2.17^{*}$ & $2.25^{*}$ & 0.04 & -1.99 & $-2.61^{* *}$ & -0.36 & 0.02 & $4.55^{* * * *}$ \\
\hline FEF.2-1.2 & $-2.35^{*}$ & 1.54 & $4.73^{* * *}$ & -0.3 & -1.23 & $-2.35^{*}$ & -1.74 & $2.7^{* * *}$
\end{tabular}


Table 7 cont $\ldots$

\begin{tabular}{lcccccccc} 
FEF25-75\% & $-3.41^{* * *}$ & $3.13^{* * *}$ & $3.13^{* * *}$ & -1.47 & -1.47 & -0.28 & $2.02^{*}$ & $2.5^{* *}$ \\
FEF75-85\% & $-4.06^{* * *}$ & $3.13^{* * *}$ & -1.54 & $-2.27^{*}$ & $-2.43^{* *}$ & -0.57 & $-2.43^{* *}$ & $3.50^{* * *}$ \\
MVV & $-2.17^{*}$ & 0.07 & $2.02^{*}$ & 1.54 & -0.43 & -1.78 & $-2.94^{* * *}$ & $2.3^{*}$ \\
PEFR & $-2.27^{*}$ & $2.10^{*}$ & $2.61^{* *}$ & 1.93 & $2.50^{* * *}$ & $2.43^{* *}$ & 0.28 & $2.89^{* * *}$ \\
\hline
\end{tabular}

$p<0.05-{ }^{*}, p<0.02-{ }^{* *}, p<0.01-{ }^{* * *}, p<0.001$ - ${ }^{* * *}$ BMl=Body Mass Index

Table 8: Percentage prevalence of respiratory abnormalities among petrol filling workers in comparison to control group

\begin{tabular}{|c|c|c|c|c|c|c|c|c|c|c|}
\hline & \multicolumn{4}{|c|}{ Petrol filling workers $(n=52)$} & & & \multicolumn{2}{|l|}{ All } & \multicolumn{2}{|c|}{ Control(n=20) } \\
\hline & \multicolumn{3}{|c|}{ Higher age group } & \multicolumn{3}{|c|}{ Lower age group } & \multirow{2}{*}{ No. } & \multirow{2}{*}{$\%$} & \multirow{2}{*}{ No. } & \multirow{2}{*}{$\%$} \\
\hline & All & $\mathbf{s}$ & NS & All & $\mathbf{S}$ & NS & & & & \\
\hline Normal & 10 & 5 & 5 & 8 & 5 & 3 & 18 & 34.61 & 17 & 85 \\
\hline Obstructive & 5 & 4 & 1 & 11 & 5 & 6 & 16 & 30.76 & 1 & 5 \\
\hline Restrictive & 8 & 5 & 3 & 4 & 1 & 3 & 12 & 23.07 & 2 & 10 \\
\hline Combined & 2 & 1 & 1 & 4 & 2 & 2 & 6 & 11.53 & 0 & 0 \\
\hline
\end{tabular}

Table 9: Prevalence of different diseases physiological symptoms among smoker and non-smoker petrol filling workers with odds ratio and $95 \%$ confidence interval.

\begin{tabular}{|c|c|c|c|c|c|c|c|c|c|c|c|c|c|c|c|c|c|c|c|c|c|}
\hline \multirow[b]{2}{*}{ GROUP } & & \multicolumn{4}{|c|}{ Cough } & \multicolumn{4}{|c|}{ Phlegm } & \multicolumn{4}{|c|}{ Chest Tightness } & \multicolumn{4}{|c|}{ Asthma } & \multicolumn{4}{|c|}{ Skin Problem } \\
\hline & & No. & $\%$ & OR & $95 \% \mathrm{Cl}$ & No. & $\%$ & OR & $95 \% \mathrm{Cl}$ & No. & $\%$ & OR & $95 \% \mathrm{Cl}$ & No. & $\%$ & OR & $95 \% \mathrm{CII}$ & No. & $\%$ & OR & $\begin{array}{c}95 \% \\
\mathrm{Cl}\end{array}$ \\
\hline S & 28 & 4 & 14.2 & 0.63 & $\begin{array}{c}0.15- \\
2.69\end{array}$ & 4 & 14.2 & 0.17 & $\begin{array}{c}0.23- \\
5.8\end{array}$ & 4 & 14.2 & 1.83 & $0.3-5.11$ & 4 & 14.2 & 1.17 & $\begin{array}{c}0.23- \\
5.8\end{array}$ & 7 & 25 & 1.26 & $\begin{array}{c}0.34- \\
4.6\end{array}$ \\
\hline NS & 24 & 5 & 20.8 & - & - & 3 & 12.5 & - & - & 2 & 8.3 & - & - & 3 & 12.5 & - & - & 52 & 20.8 & - & - \\
\hline $\mathrm{T}$ & 52 & 9 & & & & 7 & & & & 6 & & & & 7 & & & & 12 & & & \\
\hline
\end{tabular}

T: Total

Table 10: Prevalence of different physiological symptoms among higher and lower age group petrol filling workers with odds ratio and $95 \%$ confidence interval.

\begin{tabular}{|c|c|c|c|c|c|c|c|c|c|c|c|c|c|c|c|c|c|c|c|c|c|}
\hline \multirow[b]{2}{*}{ GROUP } & \multirow[b]{2}{*}{ No. } & \multicolumn{4}{|c|}{ Cough } & \multicolumn{4}{|c|}{ Phlegm } & \multicolumn{4}{|c|}{ Chest Tightness } & \multicolumn{4}{|c|}{ Asthma } & \multicolumn{4}{|c|}{ Skin Problem } \\
\hline & & No. & $\%$ & OR & $\begin{array}{c}95 \% \\
\mathrm{Cl}\end{array}$ & No. & $\%$ & OR 9 & $95 \% \mathrm{Cl}$ & I No. & $\%$ & OR & $95 \% \mathrm{Cl}$ & No. & $\% \mathrm{C}$ & OR & $\begin{array}{c}95 \% \\
\mathrm{Cl}\end{array}$ & No. & $\%$ & OR & $\begin{array}{c}95 \% \\
\mathrm{Cl}\end{array}$ \\
\hline LA & 27 & 6 & 22.2 & 0.47 & $\begin{array}{c}0.1- \\
2.1\end{array}$ & 5 & 18.5 & 0.38 & $\begin{array}{c}0.06- \\
2.18\end{array}$ & 2 & 7.4 & 2.38 & $\begin{array}{c}0.39- \\
14.3\end{array}$ & 27 & 7.43 & 3.12 & $\begin{array}{c}0.54- \\
17.8\end{array}$ & 6 & 22.2 & 1.1090 & $0.3-4.0$ \\
\hline $\mathrm{HA}$ & 25 & 3 & 12 & - & - & 2 & 8 & - & - & 4 & 16 & - & - & 52 & 20 & - & - & 6 & 24 & - & - \\
\hline $\mathrm{T}$ & 52 & 9 & & & & 7 & & & & 6 & & & & 7 & & & & 12 & & & \\
\hline
\end{tabular}

$\mathrm{LA}=$ Lower age group $\mathrm{HA}=$ Higher age group

Table 11: Prevalence of different Physiological Symptoms among petrol filling workers with different level of year of exposure with odds ratio and $95 \%$ confidence interval.

\begin{tabular}{|c|c|c|c|c|c|c|c|c|c|c|c|c|c|c|c|c|c|c|c|c|c|}
\hline \multirow{2}{*}{ years } & \multirow{2}{*}{ No. } & \multicolumn{4}{|c|}{ Cough } & \multicolumn{4}{|c|}{ Phlegm } & \multicolumn{4}{|c|}{ Chest Tightness } & \multicolumn{4}{|c|}{ Asthma } & \multicolumn{4}{|c|}{ Skin Problem } \\
\hline & & No. & $\%$ & OR & $95 \% \mathrm{Cl}$ & No. & $\%$ & OR & $95 \% \mathrm{Cl}$ & No. & $\%$ & OR & $95 \% \mathrm{Cl}$ & No. & $\%$ & OR & $95 \% \mathrm{Cl}$ & No. & $\%$ & OR & $95 \% \mathrm{Cl}$ \\
\hline$>20$ & 14 & 4 & 28.5 & 1.76 & $\begin{array}{c}0.28- \\
11.29\end{array}$ & 2 & 14.2 & 2.4 & $\begin{array}{c}0.47- \\
18.3\end{array}$ & 0 & 0 & - & - & 3 & 21.8 & 1.26 & $\begin{array}{l}0.34- \\
4.67\end{array}$ & 4 & 28.5 & 0.63 & $\begin{array}{c}0.14- \\
2.7\end{array}$ \\
\hline $11-20$ & 8 & 3 & 37.5 & 1.19 & $\begin{array}{l}0.26- \\
5.14\end{array}$ & 3 & 37.5 & 1.19 & $\begin{array}{c}0.26- \\
5.12\end{array}$ & 2 & 25 & 0.46 & $\begin{array}{c}0.06- \\
3.13\end{array}$ & 2 & 25 & 2.14 & $\begin{array}{c}0.35- \\
18.3\end{array}$ & 2 & 25 & 0.75 & $\begin{array}{l}0.12- \\
4.69\end{array}$ \\
\hline 10 & 30 & 2 & 6.6 & - & - & 2 & 6.6 & - & - & 4 & 13.3 & - & - & 2 & 6.6 & - & - & 6 & 20 & - & - \\
\hline TOTAL & 52 & 9 & & & & 7 & & & & 6 & & & & 7 & & & & 12 & & & \\
\hline
\end{tabular}




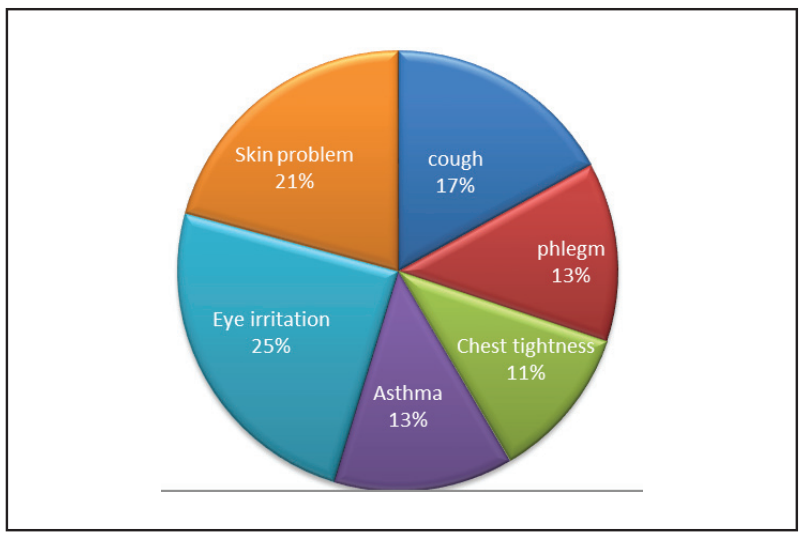

Figure 1: percentage of respiratory abnormalities among petrol filling workers.

\section{Discussion}

The major finding of this study is that there is significant decrease in FVC, FEV 1 , PEFR values when compare to their control group but the ratio of $\mathrm{FEV}_{1}, / \mathrm{FVC}\left(\mathrm{FEV}_{1 \%}\right)$ did not differ between the two groups. This finding indicates the restrictive pattern of pulmonary function in the petrol filling workers ${ }^{8}$ which corroborates with our study (PFW- $30.76 \%$ restrictive, control group 5\%). Paggiaro et al ${ }^{9}$ showed that occupational exposure to organic solvents might cause chronic air way impairment with nonspecific bronchial hyper responsiveness in shoe factory workers. Kesavachandram et al ${ }^{5}$ found that high prevalence of respiratory symptoms was primarily a consequence of exposure to the petrol vapours found in the work place in the petrol filling stations.

In the present study pulmonary function parameters SVC, TV, $\mathrm{FEF}_{25-75 \%}, \mathrm{FEF}_{75-85 \%}$, decreased significantly after 11-10 years of exposure and ERV, TV, FEF 25 75\%, $\mathrm{FEF}_{75-85 \%}$, MVV, PEFR decreased significantly after 20 years of exposure but reduction of other parameters, were not significant with the increase in year of exposure to petrol vapours. Singhal et al 10 has shown that petrol filling workers with less than 5 years had little chance to develop respiratory health problem in comparison to our study where significant lung impairment was found after 20 years of exposure. Similarly, Bhide et al ${ }^{11}$ reported that lung volumes were decreased in petrol filling workers working for more than 5 years in comparison to those working less than 5 years. Similar findings were reported by Sharma et al ${ }^{12}$ Aprajita et al ${ }^{13}$ Uzma et $\mathrm{al}^{3}$ and Dube et al. ${ }^{14}$

Exposure to diesel exhaust and other pollutants leads to respiratory symptoms and derangement in lung function. These changes are mainly due to increase in airway resistance and inflammatory changes in lungs due to exposure to diesel exhaust and petrol vapour fumes. Li et al ${ }^{15}$ reported neutrophil influx into lungs and increase in broncho alveolar lavage fluid concentration of tumor necrosis factor in rats following intratracheal instillation of ultra fine carbon particles.

Nightingale et $\mathrm{al}^{16}$ stated that Benzene content in petrol is in the range of $1-5 \%$ which has an exaggerating cause for lung function derangements in petrol filling workers.

Odds ratio of different respiratory diseases and other health problems of petrol filling workers indicated increased risk of cough, phlegm, asthma and eye irritation with the increase in years of exposure indicating that exposure to petrol and diesel exhaust for longer duration, leads to derangement of pulmonary functions. As most of petrol pumps are on heavy traffic roads, workers are also exposed to heavy air pollution. The petrol and diesel exhaust particles are very small in size about $0.2 \mathrm{~nm}$. Owing to their small size, these particles have large surface area so they can carry large amount of toxic compounds such as hydrocarbons and metals on their surface. These particles can remain air borne for longer period and can deposited deeper in smaller airways of lung ${ }^{17}$. Petrol filling workers of West Bengal do not wear personal perfective equipment so risk of development of pulmonary diseases is very high.

Comparing smoker and non smoker petrol filling workers with control group regarding pulmonary function parameters, it was found that all the pulmonary function parameters were lower in both smoker and non smoker workers than control group indicating exposure to petrol vapour is the prominent risk factor in declining the pulmonary function parameters.

Age group wise comparison of pulmonary function parameters between higher and lower age group petrol filling workers, showed that higher age group petrol filling workers had reduced pulmonary function parameters than lower age group workers indicating the effect of age in addition to petrol vapour exposure in reduction of pulmonary function parameters.

Blood pressure, resting heart rate and BMI values of petrol filling workers when compared to the control group it was found that petrol filling workers had significantly higher systolic blood pressure, heart rate, and BMI, indicating the metabolic effects of petrol vapour exposure which probably affects through $T_{3}$, $\mathrm{T}_{4}$ and TSH secretion that can influence the metabolic 
process of the body. ${ }^{3}$ Gustafson et $\mathrm{al}^{18}$ studied and found that significantly decrease in $\mathrm{TSH}$ and $\mathrm{T}_{3}$ level but $T_{4}$ levels were increasing within the normal ranges with the increase in year of exposure to petrol vapour. This decrease in TSH level could be either due to the toxic effect of solvent like benzene present in petrol vapour and polluted air. The low level of TSH is probably associated with central pituitary dysfunction due to solvent and polluted air exposure. Besides some of the solvents and pollutants like lead present in petrol vapour can accumulated in many organs including thyroid gland and exert toxic effect by causing damage directly on the target organ or depressing their functioning. ${ }^{3}$

Thus, this study demonstrates reduction of certain respiratory and physiological parameters in the occupationally exposed petrol workers. The data suggests that exposure to benzene and air pollutants may account for substantial part of respiratory and cardiovascular dysfunctions.

As benzene is the main component of petrol vapour, measurement of air quality and VOC will provide

\section{References}

1. Gupta S, Dogra TD. Air pollution and human health hazards. Indian J Occup Environ Med. 2002;6(2):8993.

2. Begam S, Ratna MB. Pulmonary function tests in petrol filling workers in Mysore city. Pak J Physiol. 2012;8(1):12-4.

3. Uzma N, Khaja BM, Salar M, Kumar BS, Aziz N, David MT, Reddy VD. Impact of organic solvents and environmental pollutants on the physiological function in petrol filling workers. Int J Environ Res Public Health. 2008;5(3):139-46

4. Badman DG, Jaffe ER. Blood and air pollution: state and knowledge and research need. Otoloryngology, Head and Neck Surg.1996;114: 205-8.

5. Kesavachandran C, Mathur N, Anand M, Dhawan A. Lung function abnormalities among petrol pump workers of Lucknow, North India. Current Science 2006; 90:1177-8.

6. The British Occupational Health Research Foundation (BOHRF). The British Occupational Health Research Foundation (BOHRF) Guidelines for Prevention, identification and Management of occupational asthma: Evidence review and recommendations. London: BOHRF; 2004. guideline for safe standard level benzene in workplace along with control strategies for air pollution.

\section{Recommendations}

Present study focuses on respiratory impairment and odds ratio indicates the increased risk of respiratory disorders of Petrol filling workers exposed to VOC. In order to prevent this risk pre employment and periodic check-up of respiratory parameters can be suggested. Besides, use of protective masks and application of engineering control system might regulate the VOC emission and working environmental can be protected. Again, participation of regular physical activity of Petrol filling workers can be recommended for increase in strength of the skeletal muscle including respiratory muscles.

Acknowledgement- Authors are thankful to Dept. of Physiology Serampore college and Dr. Anupam Bandyopadhyay, Associate Professor, Serampore College for their financial support and providing instruments for this project work. Authors are also thankful to Mr. Anay Majee, Deep learning Engineer, Intel India, for his valuable technical support.

7. American Thoracic Society (ATS) ATS- Statement Snowbird workshop on Standardization of Spirometry. Am.Rev.Resp.Dis.1979; 119,837-838

8. Sadiqua B,Rathna MB .Pulmonary function tests in Petrol filling Workers in Mysore city. Pak J Physiol. 2012;8(1):12-4.

9. Paggirao P, Lastrucci C, Di pede, Bacci E, Rossio L, Talini D. Respiratory pathology caused by exposure to solvents in shoe industry, description of 3 clinical cases. Ital Med Lav. 1985;7(4):149-52.

10. Singhal M, Khaliq F, Singhal S, Tandon OP. Pulmonary functions in petrol pump workers: a preliminary study. Indian J Physiol Pharmacol. 2007;51(3):244-8.

11. Bhide A, Munisekhar K, Hemalatha D, Gouroja SK, Pulmonary function tests in petrol pump workers in chitpur district. International Journal of Physiotherapy and Research. 2014;2(1):354-8.

12. Sharma N, Gupta N, Gupta R. Ventilatory Impaiment in petrol pump workers. JK Science. 2012;14(1):5-8.

13. Aprajita, Panwar NK, Sharma RS. A study on lung function tests in petrol pump workers. Journal of Clinical and Diagnostic Research. 2011;55:1045-50.

14. Dube S, Mungal SU, Kulkarni M. Evaluation of respiratory functions in petrol pump workers from workers at Nanded. International Journal of Recent Trends in Science and Technology. 2013;8(2):14952. 
15. Li XY, Brown D, Smith S, Mac Nee W, Donaldson K. Short term inflammatory responses following intratracheal instillation of fine and Ultra fine carbon black in rats. Inhal Toxicol. 1999;11:709-31.

16. Nightingale JA, Maggs R, Culliman P, Donnelly LE, Rogers DF, Kinnerslay R, Chung KF, Barnes PJ, Ashmore M, Taylor AN. Airway inflammation after controlled exposure to diesel exhaust particulates. American Journal of Respiratory and Critical care Medicine. 2000;162(1):161-66.

17. Levsan K. The analysis of diesel particulate. Fresenius Z Anal Chem. 1998;331:467-8.

18. Gustafson A, HerdnerP, Suchute A, Skerfving S. Occupational lead exposure and pituitary function. Int. Arch Occup. Environ Health.1989;61:277-81. 